\title{
The New Second and Higher Order Spectral Technique for Damage Monitoring of Structures and Machinery
}

\author{
Len Gelman \\ School of Engineering, Cranfield University, Cranfield, Bedfordshire, MK430AL UK. \\ L.Gelman@cranfield.ac.uk
}

\begin{abstract}
The new second and higher order spectral technique, the cross-covariance of complex spectral components, is proposed for monitoring damage of structure and machinery Normalization of the proposed technique is also developed. It is shown by simulation that the proposed technique provides effectiveness gain for detecting of damage compared to the higher order spectra.
\end{abstract}

\section{INTRODUCTION}

For monitoring damage of structure and machinery, an input excitation (e. g. vibration excitation, acoustical excitation, etc.) excites the resonance oscillations of a structure or machinery in question and resonance oscillations are processed by the higher order spectra (HOS) (Fackrell, White, Hammond, and Pinnington, 1995). This approach has been widely investigated for stationary and non-stationary signals (Kim and Powers, 1979; Fackrell et al, 1995; Collis, White, and Hammond, 1998; Schreier and Scharf, 2006; Mendel, 1991; Nikias and Mendel, 1993; McCormick and Nandi, 1999; Gelman and Petrunin, 2007; Hanssen and Scharf, 2003; Rivola and White, 1998; Gelman, White, and Hammond, 2005; Hillis, Neild, Drinkwater, and Wilcox, 2006; Hickey, Worden, Platten, Wright, and Cooper, 2009).

For diagnosing structure and machinery damage, we propose to use the normalized cross-covariance of order $n$ between $n$ complex spectral components, $n=2, \ldots, N ; N$ is the maximum value of the order of the proposed crosscovariance; there is no limitation on value $N$. The necessity and the physical sense of this proposition is that if complex spectral components have appeared due to damage, these components have non-zero cross covariance. Therefore, the proposed metric is sensitive to appearance of damage and has a clear physical sense.

Len Gelman. This is an open-access article distributed under the terms of the Creative Commons Attribution 3.0 United States License, which permits unrestricted use, distribution, and reproduction in any medium, provided the original author and source are credited.
The proposed cross-covariance could be defined in both continuous and discrete forms and, therefore, it is applicable to both continuous-time and discrete-time deterministic and stochastic signals. However, because most condition monitoring tasks are performed in the digital domain, we will highlight below the digital application of the proposed technique.

It can be easily shown that the widely used normalized HOS (Collis, White, and Hammond, 1998): i.e. the bicoherence and the skewness for the HOS of order 3 and the kurtosis and the tricoherence for the HOS of order 4, do not present the normalized cross-covariances between three and four complex spectral components respectively. It also can be shown that in the general case, the normalized HOS of order $n$ also do not present the exact normalized cross-covariances between $n$ complex spectral components. Therefore, the normalized cross- covariance between three, four and $n$ complex spectral components cannot be estimated by these traditional normalized HOS.

In addition, the proposed cross-covariance has an important advantage over the HOS: the cross-covariance is more flexible that the HOS because it can be used for any selected frequency components while the HOS are restricted in frequency component selection (Collis, White, and Hammond, 1998).

The advantage of estimating the proposed covariance in the frequency domain instead of the time domain is that localization of the frequency components of interest (e.g. resonance harmonics, etc.) could be performed.

Thus, the main novelty of this paper is the proposed new second and higher order spectral technique: the normalized cross-covariance between $n$ complex spectral components. The purposes of this paper are to:

- propose the new second and higher order spectral technique for structure and machinery health monitoring: the normalized cross-covariance of $n$ complex spectral components

- compare by simulation the proposed technique with the normalized HOS. 


\section{THE NEW HIGHER ORDER SPECTRAL CROSS- COVARIANCE}

The novel condition monitoring technique proposed here is: the normalized cross-covariance of $n$ complex spectral components. For estimating the proposed technique, the same steps should be undertaken as for estimating the HOS (Collis, White, and Hammond, 1998): the whole time domain signal should be divided into overlapped segments, $m=$ $1 . . . M, M$ defines the total number of overlapping segments in the signals. Normally, the level of the overlapping is $50 \%$; however, for a relatively small number of the non-overlapped segments for the whole signal duration (e.g. 10-20) the level of the overlapping should be increased (e.g. 60\%-80\%).

The generic expression of the proposed cross-covariance of order $n$ based on the Fourier transform is as follows:

$n \operatorname{cov}\left(f_{1}, f_{2}, \ldots, f_{n-1}\right)=\frac{1}{M} \sum_{m=1}^{M}\left\{\left[\prod_{j=1}^{n} X_{m}\left(f_{j}\right)-\bar{X}\left(f_{j}\right)\right]\right\}$

where $X_{m}\left(f_{j}\right)$ is the Fourier transform at frequency $f_{j}$ at segment duration $\Delta t_{m}$ of a signal, $j=1, \ldots, n$; $*$ is a symbol of the complex conjugate, $\bar{X}\left(f_{j}\right)$ is the mean value of variable $X\left(f_{j}\right)$.

The proposed cross-covariance (Eq. (1)) is complex valued, estimated by the Fourier transforms of a signal at $n$ frequencies and depends on $n$ frequencies. Only in the particular case of the zero-mean complex spectral components and if $f_{n}$ is the accumulated frequency of the previous frequencies, i.e. $f_{n}=\sum_{j=1}^{n-1} f_{j}$, the function (Eq. (1)) for order $n, 3$ and 4 are the classical un-normalized HOS of order $n$, the classical bispectrum and the classical trispectrum respectively. The classical bispectrum and the classical trispectrum that could be presented as follows (Collis, White, and Hammond, 1998):

$$
\begin{aligned}
& \operatorname{bisp}\left(f_{1}, f_{2}\right)=\sum_{m=1}^{M} X_{m}\left(f_{1}\right) X_{m}\left(f_{2}\right) X_{m}^{*}\left(f_{1}+f_{2}\right) \\
& \operatorname{trisp}\left(f_{1}, f_{2}, f_{3}\right) \\
& =\sum_{m=1}^{M} X_{m}\left(f_{1}\right) X_{m}\left(f_{2}\right) X_{m}\left(f_{3}\right) X_{m}^{*}\left(f_{1}+f_{2}+f_{3}\right)
\end{aligned}
$$

The classical bispectrum is a function of two frequency variables and whilst the power spectrum includes the contribution of each individual frequency component independently, the bispectrum analyses the frequency interactions between the frequency components at $f_{1}$ and $f_{2}$. The classical trispectrum is a function of three frequency variables and the trispectrum analyses the frequency interactions between the frequency components at $f_{1}, f_{2}$ and $f_{3}$. In general, the bispectrum and the trispectrum are complex quantities.

It is seen from equations of the bispectrum and the trispectrum, that these estimates are dependent on the spectral properties of a signal. Different methods are used to normalize the bispectrum and trispectrum in order to remove these dependencies. The two well-developed normalization methods for the bispectrum are the skewness and the bicoherence. Similarly, the two well-developed normalization methods for the trispectrum are the kurtosis and the tricoherence.

The bispectrum, the trispectrum and all normalized functions of the bispectrum and the trispectrum are complex valued functions. Normally, the magnitude of these functions is employed for damage diagnosis; however, the effectiveness gain could be obtained if the real and imaginary parts of the HOS are employed (Gelman, White, and Hammond, 2005). A useful feature of the bicoherence and the tricoherence is that these functions are always bounded between 0 and 1 . Values close to 0 constitute no damage case, while values close to unity constitute a severe damage.

A problem when estimating the trispectrum is to decide how to plot it. If only the bicoherence modulus is considered, it is a real function of two frequencies and can easily be plotted using the three dimensional space, where the tricoherence modulus is a function of three frequencies and, therefore, requires the four dimensional space to plot it. To achieve this plotting, a sphere should be used at each frequency point in the four dimensional space. The diameter of the sphere represents the tricoherence magnitude.

It is shown in multiple research works (e.g. Rivola and White, 1998) that the HOS is more effective for damage detection comparing with the second order signal processing techniques (e.g. the Fourier transform, the wavelet transform, etc.).

In the general case of the non-zero-mean complex spectral components, the proposed functions of order 3 and 4 are not the bispectrum and the trispectrum. The physical significance of the proposed function is that it provides a measure of the cross-covariance between $n$ complex spectral components. The proposed second and the higher order covariance in the frequency domain is an extension of the cross-covariance in time domain (Sachs, 1984) for the case of damage diagnosis. It is known from the classical statistical analysis (Sachs, 1984), that the cross-covariance in time domain should be normalized in order to avoid the misleading interpretation. The proposed cross-covariance in the frequency domain should also be normalized. The normalization allows avoidance of the misleading interpretation of the proposed technique (Eq. (1)) due to variations of the power spectral density of a signal: e.g. if power spectral density of a signal is amplified for the undamaged case, the un-normalized cross-covariance will be increased.

The standard normalization of the cross-covariance for order $n, 3$ and 4 respectively is employed here as follows:

$C\left(f_{1}, f_{2}, \ldots, f_{n-1}\right)=\frac{n \operatorname{cov}\left(f_{1}, f_{2}, \ldots, f_{n-1}\right)}{\sqrt{\left[\prod_{j=1}^{n} \operatorname{var}\left(X\left(f_{j}\right)\right)\right]}}$ 
$\operatorname{tcor}\left(f_{1}, f_{2}\right)=\frac{\frac{1}{M} \sum_{m=1}^{M} Y_{m}\left(f_{1}\right) \cdot Y_{m}\left(f_{2}\right) \cdot Y_{m}^{*}\left(f_{3}\right)}{\sqrt{\operatorname{var}\left[X\left(f_{1}\right)\right] \operatorname{var}\left[X\left(f_{2}\right)\right] \operatorname{var}\left[X\left(f_{3}\right)\right]}}$

$$
\begin{aligned}
& f \operatorname{cor}\left(f_{1}, f_{2}, f_{3}\right) \\
& =\frac{\frac{1}{M} \sum_{m=1}^{M} Y_{m}\left(f_{1}\right) \cdot Y_{m}\left(f_{2}\right) \cdot Y_{m}\left(f_{3}\right) \cdot Y_{m}^{*}\left(f_{4}\right)}{\sqrt{\operatorname{var}\left[X\left(f_{1}\right)\right] \operatorname{var}\left[X\left(f_{2}\right)\right] \operatorname{var}\left[X\left(f_{3}\right)\right] \operatorname{var}\left[X\left(f_{4}\right)\right]}}
\end{aligned}
$$

where var is the symbol of the variance,

$$
Y_{m}\left(f_{i}\right)=X_{m}\left(f_{i}\right)-\bar{X}\left(f_{i}\right) ; \quad i=1,2,3,4 \text {. }
$$

In the general case of order $n$, the normalized crosscovariance (Eq. (4)) differs from the traditional normalized double coherence (Bendat and Piersol, 1971) and the normalised HOS of order $n$ (Collis, White, and Hammond, 1998). It can be also seen from Eq. (5-6), that for orders 3 and 4 , the normalized triple and fourth covariances differs from the traditional normalized HOS, the bicoherence (Eq. 7) and skewness (Eq. 8) and the tricoherence (Eq. 9) and kurtosis (Eq. 10) respectively that could be presented as follows.

$$
\begin{aligned}
& b\left(f_{1}, f_{2}\right) \\
& =\frac{\sum_{m=1}^{M} X_{m}\left(f_{1}\right) X_{m}\left(f_{2}\right) X_{m}^{*}\left(f_{1}+f_{2}\right)}{\sqrt{\sum_{m=1}^{M}\left|X_{m}\left(f_{1}\right) X_{m}\left(f_{2}\right)\right|^{2}} \sqrt{\sum_{m=1}^{M}\left|X_{m}\left(f_{1}+f_{2}\right)\right|^{2}}}
\end{aligned}
$$

$$
=\frac{\operatorname{sk}\left(f_{1}, f_{2}\right)}{\sqrt{\sum_{m=1}^{M}\left|X_{m}\left(f_{1}\right)\right|^{2} \sum_{m=1}^{M}\left|X_{m}\left(f_{2}\right)\right|^{2} \sum_{m=1}^{M}\left|X_{m}\left(f_{1}+f_{2}\right)\right|^{2}}}
$$

$$
=\frac{t\left(f_{1}, f_{2}, f_{3}\right)}{\sqrt{\sum_{m=1}^{M}\left|X_{m}\left(f_{1}\right) X_{m}\left(f_{2}\right) X_{m}\left(f_{3}\right)\right|^{2}} \sqrt{\sum_{m=1}^{M}\left|X_{m}\left(f_{1}+f_{2+f_{3}}\right)\right|^{2}}}
$$

$$
k\left(f_{1}, f_{2}, f_{3}\right)=
$$$$
\frac{\sum_{m=1}^{M} X_{m}\left(f_{1}\right) X_{m}\left(f_{2}\right) X_{m}\left(f_{3}\right) X_{m}^{*}\left(f_{1}+f_{2}+f_{3}\right)}{\sqrt{\sum_{m=1}^{M}\left|X_{m}\left(f_{1}\right)\right|^{2} \sum_{m=1}^{M}\left|X_{m}\left(f_{2}\right)\right|^{2} \sum_{m=1}^{M}\left|X_{m}\left(f_{3}\right)\right|^{2} \sum_{m=1}^{M}\left|X_{m}\left(f_{1}+f_{2}+f_{3}\right)\right|^{2}}}
$$

This difference remains even for the zero-mean spectral components and if $f_{n}$ is the accumulated frequency of the previous frequencies, i.e. $f_{n}=\sum_{j=1}^{n-1} f_{j}$.
The proposed technique can be also used for non-stationary signals by employing the appropriate time-frequency transforms (e. g. the chirp-Wigner transform (Gelman, 2007) and the short time chirp-Fourier transform (Gelman and Ottley, 2006; etc.). This can be done by substituting the appropriate time-frequency transforms for the Fourier transform in Eq. (1), (4) - (6).

If the proposed normalized technique is being applied to monitor damaged/undamaged systems, the desirable technique ranges are as follows: magnitude values closed to 0 (i. e, no cross-covariance between the selected frequency components) constitute no damage case, while magnitude values closed to unity (i.e. high cross-covariance between the selected frequency components) constitute a severe damage.

\section{THE NUMERICAL SIMULATION}

To demonstrate that the proposed technique can effectively detect structure/machinery damage and to compare them with the traditional HOS, a simulation test with system with and without damage was performed.

An input random cosine excitation with constant amplitude, random initial phase and linearly changed instantaneous frequency in time (i.e. the chirp signal) has been passed via the following nonlinear (bilinear) system:

$\left\{\begin{array}{l}\ddot{x}+2 h \dot{x}+\omega_{s}^{2} x=A \cos \Omega(t), \quad x \geq 0 \\ \ddot{x}+2 h \dot{x}+\omega_{c}^{2} x=A \cos \Omega(t), \quad x<0\end{array}\right.$

where $x=X / m, X$ is the displacement; $h=c / 2 m, h$ is damping; $\omega_{s}=\sqrt{k_{s} / m}, \omega_{c}=\sqrt{k_{c} / m}, m$ and $c$ are the mass and damping coefficient respectively, $k_{s}$ and $k_{c}$ are the stiffness for positive displacement and the stiffness for negative displacement respectively, $A=A_{1} / m, A_{1}$ is the constant amplitude of the input signal, $\Omega(t)=\int \omega(t) d t$, $\Omega(t)$ is the instantaneous phase, $\omega(t)$ is the linearly changed angular frequency.

Equation (11) describes the system both with and without damage. For the system without damage, the stiffness for positive displacement and the stiffness for negative displacement are the same in Equation (11). For the system with damage, the stiffness for positive displacement and the stiffness for negative displacement are different in Equation (11); the level of damage severity is characterized by the stiffness ratio $k^{*}=\left(k_{c}-k_{s}\right) / k_{c}$.

The bilinear system (11) is widely used in applications, e.g. for investigation of damage of offshore structures: freehanging risers, tension leg platforms and suspended loads (Hsu, 1975; Patel, Brown and Witz, 1986; Thomson and Stewart, 1986), articulated loading towers, constrained by a connection to a massive tanker or vessels moored against fenders (Jefferys and Patel, 1982), oscillating parts with clearances and motion limiting stops (Narsiavas, 1990), 
gearboxes (Gelman, 2007), gas turbine blades (Gelman and Gorpinich, 2000) and composite materials (Brush and Adams, 2010).

System (11) describes various damage types: e.g. the "breathing" fatigue crack in structures and turbo machinery blades (Gelman and Gorpinich, 2000), pitting in gearboxes (Gelman, 2007) and damage in composite materials (Brush and Adams, 2010), etc.

Comprehensive statistics of signals were simulated in order to perform the estimation of technique effectiveness. The initial phase of the each simulated signal has been taken randomly and is uniformly distributed in the range $[0 ; 2 \pi]$.

Because the considered excitation is transient, with variable instantaneous frequency, the output signal of system (11) is also transient, with variable instantaneous frequency. It is well-known that the classical Fourier transform is not effective for processing of the transient signals; therefore, the chirp Fourier transform (Gelman and Ottley, 2006) that is suitable for the chirp signals is employed for damage detection.

The non-stationary random cosine vibration excitation excites the resonance oscillations of a system. 300 signals from system without damage (i.e. stiffness ratio is 0) and 300 signals from system with damage (i.e. stiffness ratio is nonzero) were tested for damage detection. The resonance frequencies of the systems with and without damage are 14.1 $\mathrm{Hz}$ and $13.9 \mathrm{~Hz}$ respectively, the chirp rate of the transient excitation is $0.15 \mathrm{~Hz} / \mathrm{s}$, the stiffness ratio that is characterized the relative damage size is 0.05 (i.e. the relative damage size is $5 \%$ ).

The proposed normalised cross-covariance of order 3 and the bicoherence based on the chirp-Fourier transform of the system resonance oscillations have been employed for damage detection. The cross-covariance of the fundamental, the second and the third harmonics of system resonance oscillations and the bicoherence at the fundamental and second harmonics of system resonance oscillations have been employed.

The following parameters have been used for estimating the cross-covariance of order 3 and the bicoherence in the expressions 5 and 7 respectively: in order to provide reatively good frequency resolution of the transform (i.e. $3.7 \mathrm{~Hz}$ ). the segment size is selected as $0.27 \mathrm{~s}$, the whole duration of the resonance area of the simulated signals is $5 \mathrm{~s}$ It can be estimated from the selected parameters that the number of non-overlapped segments on the whole signal duration is relatively small: i.e. 18. Therefore, segment overlapping was selected as $60 \%$. The cosine type window, the Hamming window (Oppenheim, Schafer, and Buck, 1999) is applied for each segment; the sampling frequency is $3600 \mathrm{~Hz}$.

Division of the whole time domain signal into overlapped segments is shown in Fig.1.

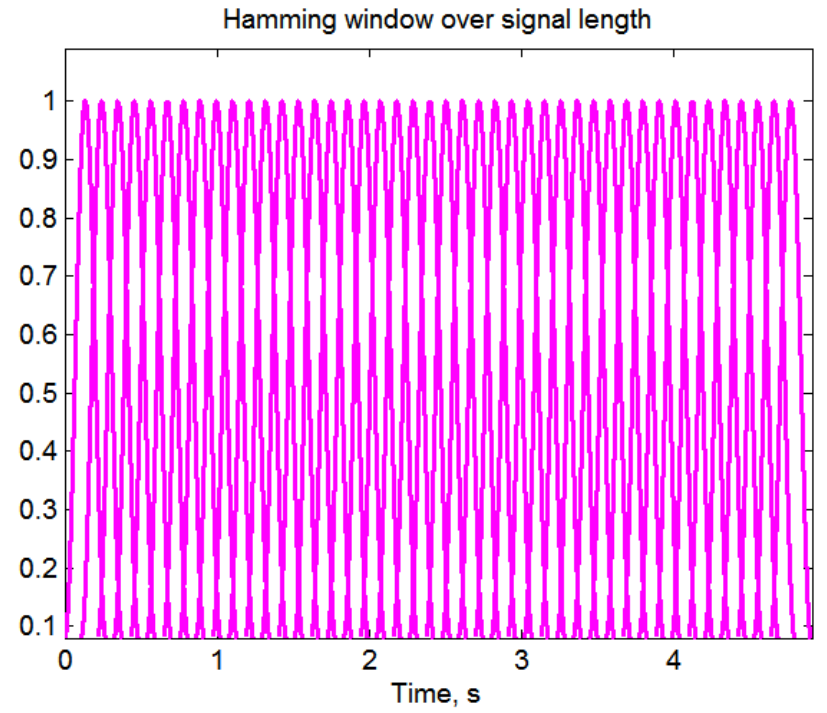

Figure 1. Division of time domain signal into overlapped segments.

The comparative effectiveness of damage detection has been estimated by the Fisher criteria (Young and Fu, 1986). The Fisher criteria for detection effectiveness are 373 and 260 for the proposed technique and the bicoherence respectively for the selected relative damage size of $5 \%$. The effectiveness comparison involves only one metric, the Fisher criterion; therefore, false alarm and missed detection rates are not estimated for this comparison.

It is known (Young and Fu, 1986) that a diagnostic feature with higher values of the Fisher criterion provides better detection effectiveness. The effectiveness gain was estimated as the ratio of the Fisher criterion for the proposed technique to the Fisher criterion for the bicoherence.

Thus, the proposed technique provides effectiveness gain 1.43 times in comparison with the bicoherence for the relative damage size of $5 \%$ and, therefore, is more effective for damage detection.

\section{Conclusions}

1. The new higher order spectral technique, the normalized cross-covariance of $n$ complex spectral components, is proposed for the first time in worldwide terms for monitoring damage in structures and machinery. Normalization of the proposed technique is also developed.

2. The proposed technique differs from the classical higher order spectral techniques. The proposed un-normalised spectral cross-covariance coincides with the classical un-normalised higher order spectra only for the particular case of the zero mean spectral components. The normalised spectral cross-covariance differs from the classical higher order spectral techniques even for the particular case of the zero mean spectral components. 
3. It is shown preliminarly by one set of simulation that the proposed technique, the third order covariance provides effectiveness gain 1.43 times for damage detection in comparison with the bicoherence. Further simulations/experiments should be performed to confirm this preliminary result.

4. The proposed technique could be extended for monitoring of damage of structure and machinery for non-stationary conditions by employing the appropriate time-frequency transforms in Eq. (1), (4)-(6).

The proposed technique could be used in mechanical and electrical engineering, telecommunication, underwater acoustics, etc.

\section{ACKNOWLEDGEMENT}

The author is very thankful to MSc student A. Stepien (Cranfield University) for carrying out a simulation and processing of the simulated data.

\section{REFERENCES}

Bendat, J. \& Piersol, A. (1971). Random data: analysis and measurement procedures. Wiley \& Sons, New York.

Brush, E. \& Adams, D. (2010). Development of a dynamic model for subsurface damage in sandwich composite materials, Proceedings of the IMAC-XXVIII, 1-4 February, Jacksonville, Florida, pp. 129-136

Collis, W. B, White, P. R, \& Hammond, J. K. (1998). High order spectra: the bispectrum and trispectrum. Mechanical Systems and Signal Processing, Vol. 12(3), pp. 375-394.

Fackrell, J. W, White, P. R, Hammond, J. K, \& Pinnington, R. J. (1995). The interpretation of the bispectra of vibration signals. Mechanical Systems and Signal Processing, Vol. 9(3), pp. 267-274.

Gelman, L. (2007). Piecewise model and estimated of damping and natural frequency for a spur gear, Mechanical Systems and Signal Processing, Vol. 21, pp. 1192-1196.

Gelman, L. (2007). Adaptive time-frequency transform for non-stationary signals with nonlinear polynomial frequency variation, Mechanical Systems and Signal Processing, Vol. 21(6), pp. 2684-2687.

Gelman, L., \& Gorpinich, S. (2000). Non-Linear Vibroacoustical Free Oscillation Method for Crack Detection and Evaluation, Mechanical Systems and Signal Processing, Vol. 14(3), pp. 343-351.

Gelman, L., \& Ottley, M. (2006). New processing techniques for transient signals with nonlinear variation of the instantaneous frequency in time, Mechanical Systems and Signal Processing, Vol. 20(5), pp. 1254-1262.

Gelman, L., \& Petrunin, I. (2007). The new multidimensional time/multi-frequency transform for higher order spectral analysis. Multidimensional Systems and Signal Processing, Vol. 18 (4), pp. 317-325.
Gelman, L., White, P., \& Hammond, J. (2005). Fatigue crack diagnostics, A comparison of the use of the complex bicoherence and its magnitude. Mechanical Systems and Signal Processing, Vol. 19(4), pp. 913-918.

Hanssen, A, \& Scharf, L. (2003). A theory of polyspectra for nonstationary stochastic processes. IEEE Transactions on Signal Processing, Vol. 51(5), pp. 1243-1252.

Hickey, D., Worden, K., Platten, M., Wright, J., \& Cooper, J. (2009). Higher-order spectra for identification of nonlinear modal coupling, Mechanical Systems and Signal Processing, Vol. 23(4), pp. 1037-1061.

Hillis, A., Neild, S., Drinkwater, B., \& Wilcox, P. (2006). Global crack detection using bispectral analysis. Proc. Royal Soc. A, Vol. 462, pp. 1515-1530.

Hsu, C. (1975). The response of a parametrically excited hanging string in fluid. ASME Journal of Sound and Vibration, Vol. 39, pp 305-316.

Jefferys, E. \& Patel, M. (1982). Dynamic analysis models of the tension leg platform. ASME Journal of Energy Resources Technology, Vol 104, pp 317-323.

Kim, Y. C., \& Powers, E. J. (1979). Digital bispectral analysis and its applications to non-linear wave interactions. IEEE Transactions on Plasma Science, Vol. 7(2), pp. 120-131.

McCormick, A. C., \& Nandi, A. K. (1999). Bispectral and trispectral features for machine condition diagnosis. IEE Proc. of the Vision, Image and Signal Processing, Vol. 146 (5), pp. 229-234.

Mendel, J. M. (1991). Tutorial on higher-order statistics (spectra) in signal processing and system theory: theoretical results and some applications. Proc. of the IEEE, Vol. 79 (3), pp. 278-305.

Narsiavas, S. (1990). On the dynamics of oscillators with bilinear damping and stiffness. International Journal of Non-linear Mechanics, Vol 25(5), pp 535-554.

Nikias, C. L, \& Mendel, J. M. (1993). Signal processing with higher-order spectra. IEEE Signal Processing Magazine, Vol. 10 (3), pp. 10-38.

Oppenheim A., Schafer, R., \& Buck, J. (1999). Discrete-Time Signal Processing. Upper Saddle River, NJ: Prentice Hall, pp. 468.

Patel, M., Brown, D. \& Witz, J. (1986). Operability analysis for a mono-hull crane barge. Proc. of the RINA, spring meeting.

Rivola, A., \& White, P. (1998). Detecting system nonlinearities by means of higher order statistics. Proceedings of the 3rd International Conference on Acoustical and Vibratory Surveillance Methods and Diagnostic Techniques, 13-15/10/1998, Senlis, France, Vol. 1, pp. 263-272.

Sachs, L. (1984). Applied Statistics - A Handbook of Techniques, Springer-Verlag, New York.

Schreier, P. J, \& Scharf, L. L. (2006). Higher-order spectral analysis of complex signals. Signal Processing, Vol. 86 (11), pp. 3321-3333. 
Thomson, J. \& Stewart, H. (1986). Nonlinear dynamics and chaos. John Willey.

Young, T., \& Fu, K. S. (1986). Handbook of pattern recognition and image processing. New York, Academic. 